\title{
Fabrication of Blue GaN Light-Emitting Diodes by Laser Etching
}

\author{
Fu-Hsiang YANG, Chao-Jen HsiaO, Ying-Jay YANG, Jun-Hong LIN ${ }^{1}$ and Lon WANG ${ }^{1}$ \\ Department of Electrical Engineering, National Taiwan University, Taipei, Taiwan, R.O.C. \\ ${ }^{1}$ Institute of Optoelectronic Engineering, National Taiwan University, Taipei, Taiwan, R.O.C. \\ (Received January 15, 2002; accepted for publication February 12, 2002)
}

GaN light-emitting diodes (LEDs) were fabricated using a $248 \mathrm{~nm} \mathrm{KrF}$ excimer laser. Thick photoresist was used to form an etching mask to resist the strong laser irradiation and the unmasked top p-type GaN layer was removed effectively to reveal the bottom n-type GaN layer for the n-type contact. The etching rate of GaN film by laser irradiation and the morphology of the etched surface were studied by atomic force microscopy (AFM). Current-voltage ( $I-V)$ and photoluminescence (PL) characteristics of GaN LEDs fabricated by laser etching were measured and compared to those of LEDs fabricated by commercial inductively coupled plasma (ICP) etching. The performance of GaN LEDs fabricated by laser etching was comparable to that of LEDs fabricated by commercial ICP etching. [DOI: 10.1143/JJAP.41.L468]

KEYWORDS: GaN, LED, KrF excimer laser, ICP, laser etching

GaN-based semiconductors have become the most important materials for short-wavelength optoelectronic devices. In addition, the wide band gap, high dielectric strength and high thermal conductivity make GaN a great choice of semiconductor for microwave power amplifiers in high-temperature and high-power environments. For the efficient fabrication of GaN-based devices, dry etching technology is essential because GaN resists conventional wet etching due to its inertness to chemical solutions. Dry etching technologies such as inductively coupled plasma (ICP), ${ }^{1)}$ reactive ion etching $(\mathrm{RIE})^{2)}$ and chemical-assisted ion beam etching $(\mathrm{CAIBE})^{3)}$ are effective for patterning $\mathrm{GaN}$ and have been developed into commercial systems. An alternative method using deep UV pulsed laser ablation was demonstrated to achieve deep etching of GaN. ${ }^{4,5)}$ Well-defined patterns of GaN were realized by thermal decomposition of $\mathrm{GaN}$ into nitrogen and gallium droplets using deep UV laser irradiation. In this study, we report for the first time the fabrication of blue GaN lightemitting diodes (LEDs) whose mesa were created by the etching of a $\mathrm{KrF}(248 \mathrm{~nm})$ pulsed excimer laser. Instead of the commonly used metal mask, we chose a thick photoresist pattern as the etching mask to define the pattern of light-emitting diode (LED) mesas. The thick photoresist shows very good resistance to the strong laser irradiation. The characteristics of blue GaN LEDs were measured and showed performance as good as that of LEDs fabricated by commercially available ICP etching.

The GaN LED wafers were grown by the organometallic vapor-phase epitaxy (OMVPE) method. Sapphire with (0001) orientation was used as the substrate. The LED structure consists of a $30 \mathrm{~nm}$ low-temperature buffer layer, a $3 \mu \mathrm{m}$ layer of Si-doped n-type GaN, an active region consisting of five pairs of $4 \mathrm{~nm} \mathrm{In}_{0.2} \mathrm{Ga}_{0.8} \mathrm{~N}$ well layers sandwiched by $8 \mathrm{~nm} \mathrm{GaN}$ barrier layers, a $10 \mathrm{~nm}$ layer of $\mathrm{Mg}$-doped p-type $\mathrm{AlGaN}$, and a $300 \mathrm{~nm}$ layer of $\mathrm{Mg}$-doped p-type $\mathrm{GaN}$.

A $248 \mathrm{~nm} \mathrm{KrF}$ excimer laser was used in our experiment to selectively remove the unmasked top p-type layer to expose the bottom n-type $\mathrm{GaN}$ layer for the n-type contact as realized by dry etching in conventional GaN LEDs. The pulse duration is $20 \mathrm{~ns}$ with a repetition rate of $1 \mathrm{~Hz}$ and the energy density of each laser pulse ranges from 200 to $800 \mathrm{~mJ} / \mathrm{cm}^{2}$. The MicroChem negative photoresist SU-8 25 was chosen as the etching mask because it can form a thick film. The pho- toresist was applied to the wafer and spin coated with a spin speed of $3000 \mathrm{rpm}$ for $40 \mathrm{~s}$, resulting in a $20 \mu \mathrm{m}$-thick film. The pattern was formed by exposing with i-line Karr-Suss and then developed with a MicroChem SU-8 developer. The laser etching was conducted in atmosphere at room temperature. To achieve smooth morphology of the ecthed surface and a linear etching rate corresponding to the number of pulses, the wafer has to be dipped into $\mathrm{HCl}$ solution to remove $\mathrm{Ga}$ droplets on the surface after each pulse. After laser etching, the photoresist looked clear and was in good condition except for a sputtered photoresist that was a few micrometers thick. Finally, the photoresist was removed by a MicroChem Remover PG.

Mesas $600 \mathrm{~nm}$ high were realized after shooting twelve laser pulses with an energy density of $600 \mathrm{~mJ} / \mathrm{cm}^{2}$ on the unmasked p-type GaN layer and the bottom n-type GaN layer was exposed. Next, a thin $\mathrm{Ni}$ and Au films (6 nm and $5 \mathrm{~nm}$ in thick, respectively) were evaporated onto the p-type $\mathrm{GaN}$ layer as a transparent p-electrode. Then, a Ni/Au p-bonding pad and a Ti/Al contact were evaporated onto the p-type and n-type GaN layers, respectively. The wafer was cut into small dice that were then bonded on TO-cans for measurement.

For comparison, the same wafer was used to fabricate LEDs by a commercial ICP etching system. The roughness of the laser-etched $\mathrm{GaN}$ surface was measured with atomic force microscopy (AFM) and the output characteristics of both types of LEDs were measured under DC-biased conditions.

Figure 1 shows the relationship of the etching rate the energy density of a single laser pulse. The threshold energy density for the etching is about $300 \mathrm{~mJ} / \mathrm{cm}^{2}$. With an energy density of $800 \mathrm{~mJ} / \mathrm{cm}^{2}$, an etching rate of $65 \mathrm{~nm}$ per pulse can be achieved. A slow saturation of the etching was observed due to the exponential decay of the laser light into the film. This phenomenon is evidence of behavior following BeerLambert's law and was also observed in other experiments. ${ }^{6}$ )

Initially, as in many other experiments on laser ablation, we also attempted using several refractory metals as the mask materials. However, the metal films used were mostly evaporated even after the irradiation of only a few successive laser pulses. ${ }^{7,8)}$ As an alternative, we found that a thick photoresist mask showed better ablation properties under the laser irradiation. After $6-8$ shots of laser pulse, only $6 \mu \mathrm{m}$ of a $20-\mu$ m-thick photoresist was removed. The mechanism is dis- 


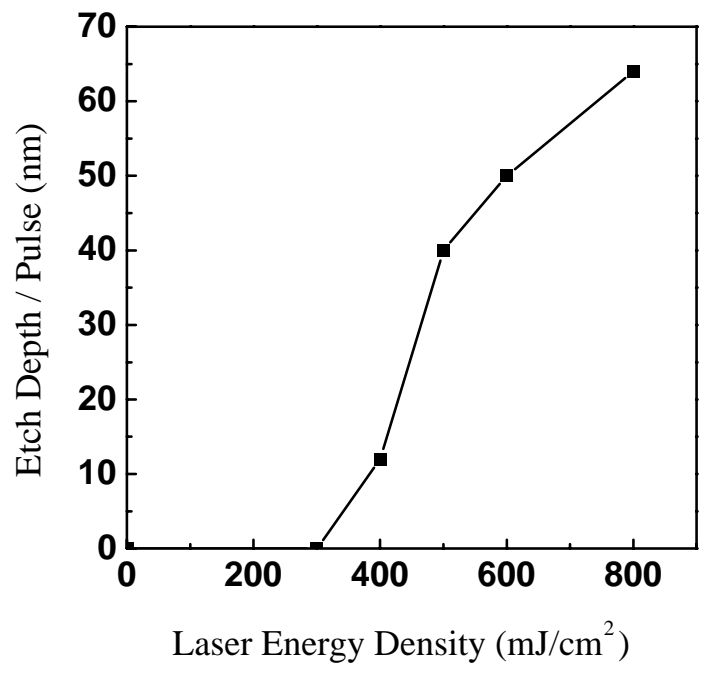

Fig. 1. Dependence of the etch rate on incident laser fluence. The threshold for etching is about $300 \mathrm{~mJ} / \mathrm{cm}^{2}$.

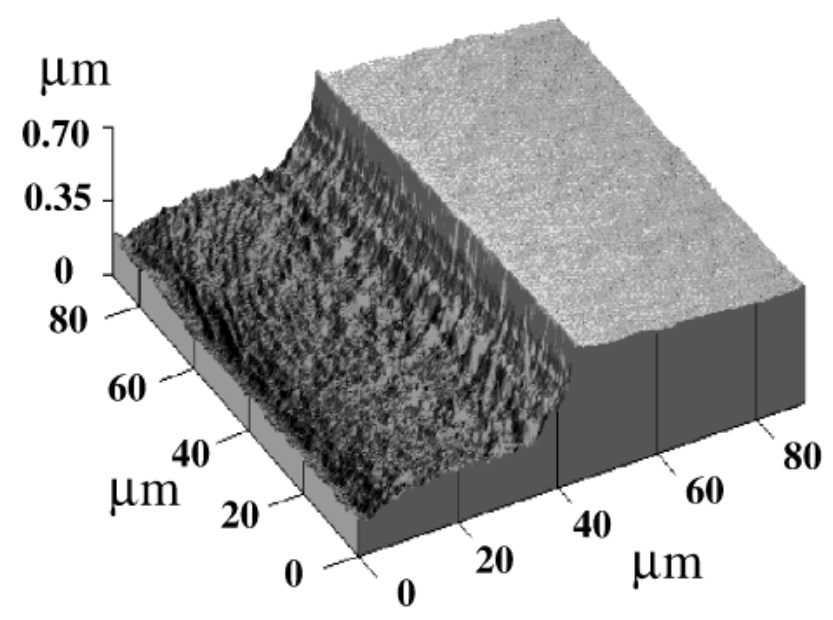

Fig. 2. AFM image of a laser-etched step with a height of about $0.5 \mu \mathrm{m}$.

cussed below. The absorption coefficient of photoresist used is $10^{4}-10^{5} \mathrm{~cm}^{-1}$, so that the laser penetration depth is estimated to be less than $1 \mu \mathrm{m}$. Since the thermal conductivity of the photoresist is much lower than that of metals, the heat generated by the laser pulse will be localized within a thin surface layer, resulting in layer-by-layer ablation. On the other hand, the heat generated in the metal mask by the laser pulse will spread throughout the entire mask rapidly and most of the metal film will be evaporated or distorted.

Figure 2 shows the AFM image of the laser-etched surface. The root-mean-square roughness was about $15 \mathrm{~nm}$, which was larger than that with ICP etching. The roughness may be caused by the masking effect of the Ga droplets that aggregated nonuniformly during each laser pulsation, which were observed by optical microscopy and AFM. However, the etched surface still looked flat and specular under optical microscope. Figure 3 shows the $I-V$ and $L-I$ characteristics of both GaN LEDs fabricated by the laser etching and by commercial ICP etching. One can see that the characteristics of the two LEDs are almost identical. The differential resistance of the two LEDs was about $30 \mathrm{ohm}$. The light outputs of both LEDs saturated at high injection current mainly due to the thermal heating. Both efficiencies were about $3 \%$,

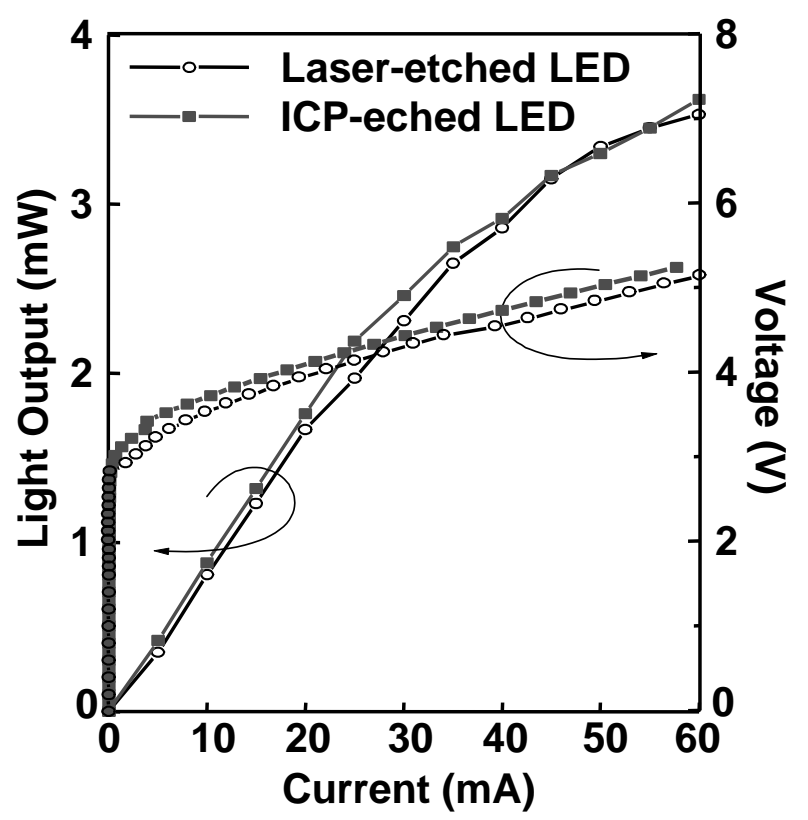

Fig. 3. $L-I$ and $V-I$ characteristics of two blue GaN LEDs fabricated by laser and ICP etching. The devices show a comparable performance.

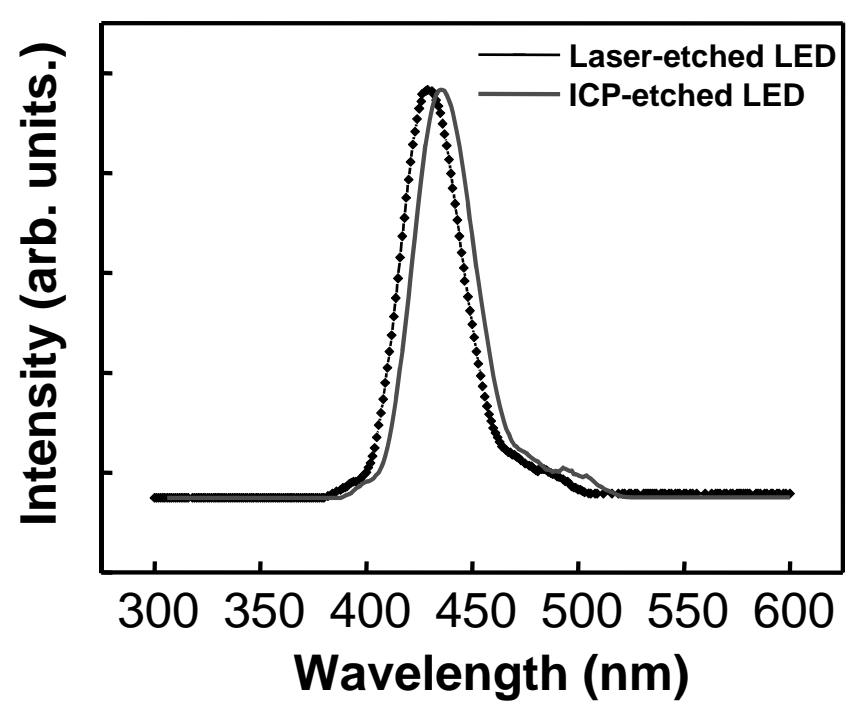

Fig. 4. EL spectra of the same two blue GaN LEDs as shown in Fig. 3. The slight shift of the two spectra is due to the nonuniformity of the wafer.

which is comparable to the previously published results. ${ }^{9)}$ Figure 4 shows optical spectra of the same two GaN LEDs. The emission wavelength was centered at around $430 \mathrm{~nm}$ with a width of $30 \mathrm{~nm}$. No deterioration of the output characteristics was observed for the LEDs fabricated by laser etching. The slight shift of the spectra between the two LEDs was due to the nonuniformity of the wafer. The blue GaN LEDs fabricated by the laser etching and commercial ICP etching respectively exhibited almost identical performances, indicating that the laser etching technology is a good alternative method for $\mathrm{GaN}$ device fabrication. In particular, laser etching can be carried out in air instead of under vacuum conditions and generates no chemical waste, making it a great choice for the high throughput and low-environment-impact production of nitride-based devices.

We have demonstrated for the first time that blue GaN 
LEDs can be fabricated by laser etching. A $248 \mathrm{~nm} \mathrm{KrF} \mathrm{ex-}$ cimer laser was used for the $\mathrm{GaN}$ mesa etching and a thick photoresist mask was used to resist the strong laser etching. The performance of blue GaN LEDs fabricated by the laser etching was as good as that of those fabricated by commercial ICP etching, indicating that laser etching is a good alternative for the fabrication of GaN-based devices. The fact that there is no need to carry out the laser etching under vacuum conditions and that no chemical waste is generated also make the process even more attractive in terms of mass production and low environmental impact.

1) R. J. Shul, G. B. McClellan, S. A. Casalnuovo, D. J. Rieger, S. J. Pearton,
C. Constantine, C. Barratt, R. F. Karlicek, Jr., C. Tran and M. Schurman: Appl. Phys. Lett. 69 (1996) 1119.

2) I. Adesida, A. Mahajan, E. Andideh, M. A. Kahn, D. T. Olson and J. N. Kuznia: Appl. Phys. Lett. 63 (1993) 2777.

3) A. T. Ping, I. Adesida and M. A. Khan: Appl. Phys. Lett. 67 (1995) 1250.

4) J. Zhang, K. Sugioka, S. Wada, H. Tashrio and K. Midorikawa: J. Cryst. Growth 189/190 (1998) 725.

5) M. K. Kelly, O. Ambacher, B. Dahlheimer, G. Groos, R. Dimitrov, H. Angerer and M. Stutzmann: Appl. Phys. Lett. 69 (1996) 1749.

6) A. M. Dhote, R. Shreekala, S. I. Patil, S. B. Ogale, T. Venkatesan and C. M. Williams: Appl. Phys. Lett. 67 (1995) 3644.

7) S. K. Lee, W. S. Chang and S. J. Na: J. Appl. Phys. 86 (1999) 4282.

8) J. R. Ho, C. P. Grigoropoulos and J. A. C. Humphrey: J. Appl. Phys. 78 (1995) 4696.

9) S. Nakamura, S. Pearton and G. Fasol: The Blue Laser Diode (Springer, New York, 2000) 2nd ed., Chap. 9, p. 198. 